\title{
Suporte de Leitura: desenvolvimento de um protótipo para auxílio a pessoas com baixa visão - testes preliminares
}

\section{Reading Support: development of a prototype for aiding people with low vision - preliminary tests}

\author{
Fernanda M. Rodrigues M. Ferreira ${ }^{1}$, Marcos Vinícius Bortolus ${ }^{2}$, Áquila Loran \\ Nolasco $^{3}$, Emanuel Reis de Melo ${ }^{4}$, Mariana Borges Viotti ${ }^{5}$, Renata M. Lopes ${ }^{6}$, \\ Adriana Maria Valladão Novais Van Petten ${ }^{7}$
}

http://dx.doi.org/10.11606/issn.2238-6149.v26i2p288-293

\begin{abstract}
Ferreira FMRM, Bortolus MV, Nolasco AL, Melo ER, Viotti MB, Lopes RM, Van Petten AMVN. Suporte de leitura: desenvolvimento de um protótipo para auxílio a pessoas com baixa visão - testes preliminares. Rev Ter Ocup Univ São Paulo. 2015 maio-ago.;26(2):288-93.
\end{abstract}

RESUMO: A baixa visão é uma condição intermediária entre a visão normal e a cegueira, secundária a um acometimento irreversível do sistema visual, acarretando prejuízos no desempenho e na realização de determinadas atividades. Para minimizar esses problemas, é recomendada a utilização dos recursos de Tecnologia Assistiva. O objetivo deste trabalho foi fazer um relato do desenvolvimento de um protótipo de Suporte de Leitura (SL) e descrever os resultados dos testes preliminares. Quanto ao aspecto metodológico, foi feito um levantamento dos diferentes dispositivos de auxílio para indivíduos com baixa visão disponíveis no mercado e definiu-se pela construção do protótipo do Suporte de Leitura, que contemplasse as necessidades ergonômicas do usuário, com baixo custo, de fácil acesso, dentro do conceito de design universal. Os resultados obtidos foram: suporte de leitura composto por uma base de sustentação, um tampo reclinável facilitando a portabilidade e trilhos para movimentação do suporte da lente. Concluiu-se que o equipamento desenvolvido possui aspectos positivos, como melhor manutenção da postura, boa usabilidade, portabilidade considerável e boa viabilidade ergonômica. Contudo será fundamental dar continuidade aos testes do equipamento com a população-alvo para garantir a usabilidade e aplicabilidade reais do dispositivo.

DESCRITORES: Tecnologia educacional; Baixa visão; Desenho de equipamento; Tecnologia de baixo custo.

Ferreira FMRM, Bortolus MV, Nolasco AL, Melo ER, Viotti MB, Lopes RM, Van Petten AMVN. Reading Support: development of a prototype for aiding people with low vision - preliminary tests. RevTerOcupUniv SãoPaulo.2015 May-Aug.;26(2):288-93.

\begin{abstract}
Low vision is an intermediate condition between normal vision and blindness, secondary to an irreversible impairment of the visual system, causing losses in performance and achievement of certain activities. To minimize these problems, it is recommended the use of Assistive Technology resources. The objective of this paper was to report the development of a Reading Support (RS) prototype and describe the results of the preliminary tests. For the methodological aspect, a survey of different assistive devices available for individuals with low vision was conducted and it was decided to build a Reading Support prototype that considers the ergonomic needs of the user, with low cost, easy access and within the concept of universal design. The results were: reading support comprised of a support base, a reclining top facilitating the portability and rails to drive
\end{abstract}

1. Graduada em Terapia Ocupacional pela Universidade Federal de Minas Gerais UFMG, Belo Horizonte, Brasil.

2. Professor Adjunto do Departamento de Engenharia Mecânica da UFMG; Coordenador do projeto de extensão PARAMEC da

Universidade Federal de Minas Gerais, Belo Horizonte, MG, Brasil. E-mail: borta@demec.ufmg.br

3. Aluno de graduação em Engenharia Civil da Universidade Federal de Minas Gerais, Belo Horizonte, MG, Brasil.

4. Aluno de graduação em Engenharia Mecânica da Universidade Federal de Minas Gerais, Belo Horizonte, MG, Brasil.

5. Aluno de graduação em Terapia Ocupacional da Universidade Federal de Minas Gerais, Belo Horizonte, MG, Brasil

6. Graduada em Terapia Ocupacional pela Universidade Federal de Minas Gerais, Belo Horizonte, Brasil.

7. Professora Adjunta do Departamento de Terapia Ocupacional, Universidade Federal de Minas Gerais, Belo Horizonte, MG, Brasil.

Autor para correspondência: Fernanda Márcia Rodrigues Martins Ferreira. Universidade Federal de Minas Gerais, Galpão do Departamento de Engenharia Mecânica - DEMEC, sala 1900. Av. Antônio Carlos, 6627, Pampulha, Belo Horizonte, MG, Brasil. CEP: $31270-901$. e-mail: nanda_rodrigues06@hotmail.com. 
the lens holder. It was concluded that the equipment developed has positive aspects such as better maintenance of posture, good usability, considerable portability and good ergonomic viability, but it will be essential to continue the equipment testing with the target population to ensure the actual usability and applicability of the device.

KEYWORDS: Educational technology; Low vision; Equipment design; Low cost technology.

da visão de cores e da adaptação à luminosidade ${ }^{7,8,9}$. Estes acometimentos apresentam grave repercussão sobre a vida do indivíduo, em qualquer faixa etária, causando limitações em atividades do dia a dia, dentre elas: identificar cores e peças do vestuário, consultar relógio, identificar chamadas telefônicas, cozinhar, ter mobilidade independente, ler, trabalhar, escrever, afetando sobremaneira seu desempenho ocupacional e qualidade de vida ${ }^{10}$.

A baixa visão também tem impacto na educação, constituindo importante causa de limitação na idade escolar, tendo em vista o processo de ensinoaprendizagem, comprometendo, além da escrita e leitura, a interação social e consequentemente a inclusão escolar ${ }^{11}$. Crianças com baixa visão, por possuírem algumas limitações quanto às possibilidades de locomoção e exploração de objetos, são frequentemente consideradas incapazes de executarem certas atividades, de participarem, decidirem por si mesmas, contribuírem em atividades realizadas em grupo e em brincadeiras livres, havendo subestimação de suas potencialidades e capacidades, interferindo assim na participação e integração junto aos demais colegas de turma e professores, dificultando a inclusão escolar ${ }^{12}$.

Para minimizar esses problemas, melhorar as condições de aprendizagem, o desempenho visual e o rendimento global de crianças em idade escolar com baixa visão é recomendada a utilização dos recursos de Tecnologia Assistiva (TA) ${ }^{13}$. De acordo com Gasparetto ${ }^{14}$, tecnologia assistiva é a área do conhecimento, de característica interdisciplinar, que engloba produtos, recursos, metodologias, estratégias, práticas e serviços que objetivam promover a funcionalidade, relacionada à atividade $\mathrm{e}$ participação, de pessoas com deficiência, incapacidades ou mobilidade reduzida, visando a sua autonomia, independência, qualidade de vida e inclusão social.

Atualmente, o mercado oferece recursos de TA específicos que potencializam o funcionamento visual do indivíduo em suas atividades diárias, classificados como auxílios ópticos (lupas, sistemas telescópicos, réguas de leitura e óculos especiais), não ópticos (luminárias, apoio de livros e pranchas de leitura), eletrônicos (lupa eletrônica, detector de cores colorido, calculadora sonora) e de informática (teclado ampliado, comunicação 
tátil: Braille e Tinta, impressão ampliada $)^{15,16}$. Em paralelo a essa oferta de recursos, existe uma demanda crescente por equipamentos que potencializem o funcionamento visual do deficiente visual associado a aspectos ergonômicos, já que eles constituem-se numa população cada vez mais leitora, exigente, ativa e numerosa nos dias atuais, que abrange desde os jovens até os idosos ${ }^{17}$. Entretanto observa-se uma carência de investimentos e estudos nesta área, o custo elevado dos equipamentos hoje disponíveis no mercado, ópticos ou não, problemas relacionados ao aspecto ergonômico do dispositivo, que exige, muitas vezes, a manutenção de posturas inadequadas por parte do usuário na realização das tarefas, gerando fadiga muscular; a dificuldade para escrever ao utilizar-se as lupas manuais ou de apoio ${ }^{6}$. Nota-se, portanto, a necessidade de desenvolver novas estratégias e equipamentos de tecnologia assistiva que avancem no atendimento às questões ergonômica, econômica e de acessibilidade.

Em tal contexto, este trabalho tem o objetivo de descrever o desenvolvimento do protótipo de Suporte de Leitura (SL) e os resultados dos testes preliminares.

\section{METODOLOGIA}

O dispositivo de tecnologia assistiva (SL) foi desenvolvido pelo PARAMEC, grupo de trabalho multidisciplinar, composto por docentes da Universidade Federal de Minas Gerais (UFMG) e discentes de graduação, que tem como missão projetar soluções de baixo custo que atendam às demandas de pessoas com deficiências que apresentam dificuldades para a realização de suas atividades cotidianas.

As etapas do projeto estão apresentadas na Figura 1.

A definição da construção de um equipamento para baixa visão partiu de uma solicitação de uma escola de ensino fundamental ao PARAMEC. A partir dessa demanda, o grupo fez visitas à escola para conhecer sua realidade física, humana e organizacional e detectar a especificidade do DTA. A partir da observação in loco, o grupo definiu o tipo de DTA (SL) a ser realizado, bem como o subgrupo responsável pelo seu desenvolvimento.

Foi realizado o levantamento bibliográfico sobre DTAs para baixa visão, especificamente para potencializar a leitura, bem como um levantamento de mercado e revendedores que comercializam esses dispositivos para subsidiar uma avaliação estética e funcional dos mesmos. A partir da revisão bibliográfica e informações do mercado foi elaborado um anteprojeto do DTA proposto (SL), assim como o levantamento do material adequado a sua construção e orçamento.

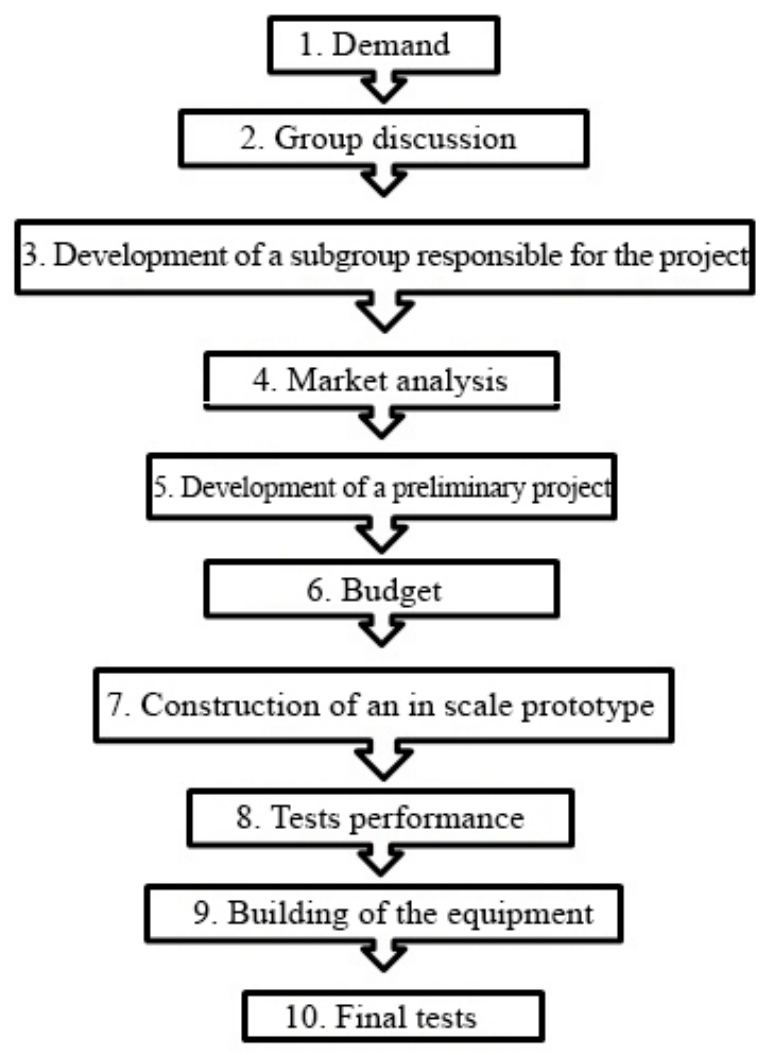

Figura 1 - Etapas de desenvolvimento do projeto

De posse dos materiais e projetos de construção, o grupo PARAMEC estabeleceu uma parceria com o Colégio Técnico da UFMG (Coltec) para a fabricação do primeiro protótipo do suporte. Utilizando a infraestrutura do Coltec foram realizadas as etapas de construção, envolvendo cortes, soldagem e montagem final do equipamento. O software 3D CAD Design SolidWorks foi usado para a concepção das peças do projeto e o desenho técnico. O protótipo foi confeccionado em escala e submetido a testes preliminares como inclinação do tampo adequada para uma postura correta, funcionalidades mecânicas, posicionamento da lente e movimentação da mesma.

Em reuniões semanais do grupo PARAMEC, foram propostas adaptações, melhorias e novas abordagens para a resolução dos problemas que apareceram durante o desenvolvimento do protótipo, de forma a melhorar suas condições de utilização, reduzindo custos de fabricação e de materiais utilizados. Após a confecção do protótipo, o equipamento foi utilizado pelos próprios membros da equipe com o objetivo de avaliar a inclinação adequada para o material de leitura, a segurança, bem como a usabilidade do SL. 


\section{RESULTADOS}

O Suporte de Leitura (SL) foi desenvolvido com o intuito de facilitar o acesso a leituras de rotina e o aprendizado de estudantes no ambiente escolar, proporcionando maior inclusão e melhor aproveitamento educacional. Foi desenvolvido para atender às necessidades de alunos com baixa visão, compreendendo a maior parte da população brasileira, dentro do conceito de design universal, com possibilidade de ser empregado em várias instituições, com um acesso livre e de baixo custo.

A partir da revisão da literatura e da análise de mercado, foram levantados alguns aspectos ergonômicos e mecânicos que poderiam ser melhorados nos equipamentos hoje disponíveis no mercado. Essa análise mostrou dois equipamentos de suporte de leitura que serviram como base para este estudo. O primeiro foi a Prancha de Leitura feita de PVC (policloreto de vinila) e perfis de alumínio. Ela possui lente de aumento e bolsa acoplada de armazenamento de protótipo. "A prancha apresenta, na sua parte de trás, local de encaixe de um apoio, para permitir a sua inclinação de $45^{\circ}$ se o usuário assim o desejar e uma elevação na sua parte inferior para apoio do material de leitura e/ou escrita, com o limite máximo da espessura pré-estabelecido em $4 \mathrm{~cm}$ de lombada" ${ }^{\text {. O }}$ segundo dispositivo foi a prancha de leitura produzida pela Bonavision, empresa científica do Núcleo de Empresas e Empreendimentos Tecnológicos Inovadores na USP (Cietec), que desenvolve equipamentos inovadores para ampliação da imagem. Esse equipamento já possui mercado nacional consolidado. Possui uma lupa de 22 graus positivos (dioptrias), cinco centímetros de diâmetro, aumento de seis vezes, com ajuste de foco individualizado. Acoplada à prancha de leitura, a lupa desliza horizontalmente em trilhos, para acompanhar a linha de texto a ser lido. A posição inclinada da prancha permite ao usuário olhar pela lupa sem inclinar em demasia o pescoço ${ }^{18}$.

As pranchas de leitura apresentadas encontramse no mercado a um preço inacessível para usuários de baixa condição socioeconômica. Ambas possuem graus de inclinação reduzidos, dificultando a manutenção de uma postura adequada para leitura ${ }^{19}$. Considerando esses aspectos, o anteprojeto de equipamento de leitura (SL) buscou atender às seguintes condições: baixo custo, melhor condição postural para leitura, peso e resistência, portabilidade, usabilidade, adaptável a qualquer ambiente, boas condições de operação na área escolar e as necessidades do usuário.

O projeto resultou na confecção de três protótipos, em função das diversas necessidades de adaptação. A versão 1.3 do SL está apresentada na Figura 2.

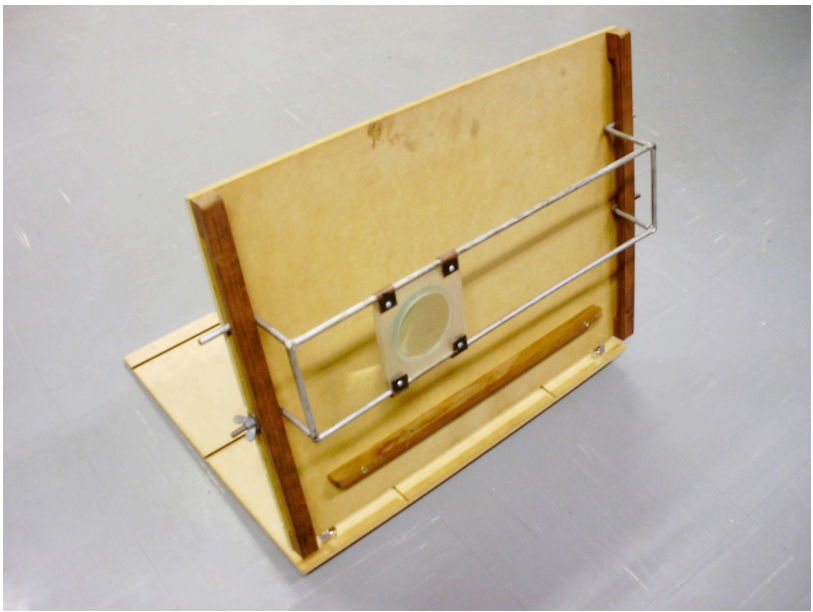

Figura 2 - SL versão 1.3

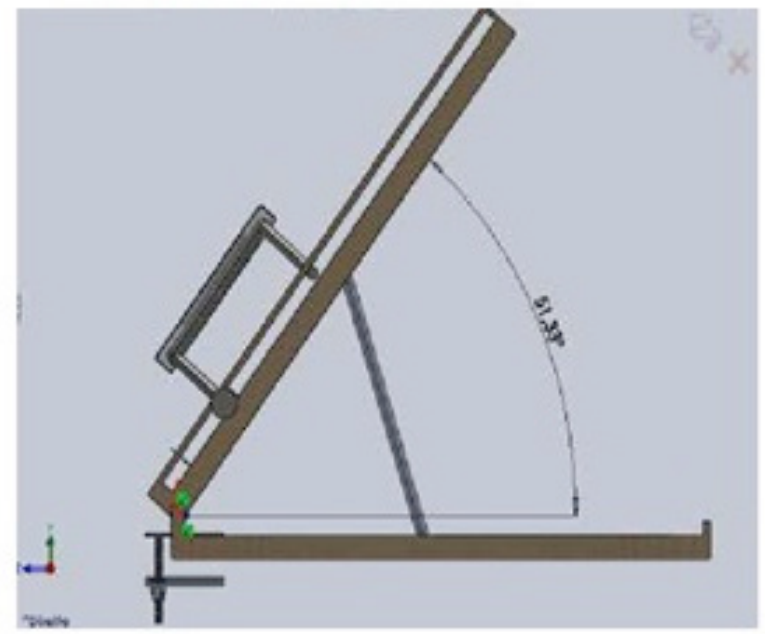

Figura 3 - Vista Lateral do suporte com inclinação de $51^{\circ}$

O SL é composto por uma base de sustentação, um tampo reclinável e trilhos para movimentação do suporte da lente. As dimensões foram adotadas considerando o comprimento e a largura dos materiais de leitura utilizados pelo usuário, como cadernos, livros e revistas.

A inclinação do dispositivo foi obtida por meio de uma haste apoiada entre o tampo e a base do suporte. $\mathrm{Na}$ parte posterior do tampo foram confeccionadas três extrusões passantes, que fornecem as inclinações de $80^{\circ}$, $65^{\circ}$ e $51^{\circ}$ do tampo em relação à base do suporte.

O material usado para sua construção (base) foi o Medium Density Fiberboard (MDF), em razão da facilidade de usinagem, peso, custo de mercado e estabilidade dimensional devido à umidade, o que facilita sua higienização. 
Com relação às questões ergonômicas, os testes preliminares realizados pela própria equipe de pesquisa sugerem as seguintes vantagens: as três inclinações favoreceram a manutenção de uma postura mais adequada do usuário (Figura 4). Além disso, a dimensão do SL permitiu a utilização de livros de diferentes brochuras, inclusive livros infantis, que geralmente são de tamanhos maiores; a lente pode ser movimentada sobre o tampo nas direções vertical e horizontal, podendo fixar a direção vertical, o que impede que a linha de leitura seja prejudicada por fatores adversos; o tampo pode ser totalmente reclinável, facilitando a portabilidade.

Os testes preliminares indicaram aspectos bastante positivos do dispositivo, tais como: melhor manutenção da postura, boa usabilidade, portabilidade considerável e ergonomicamente viável. No entanto serão necessários novos testes de laboratório para avaliar o acoplamento de iluminação, elaboração de um encaixe universal para lente de aumento e um melhor estudo do material. Para garantir a usabilidade e aplicabilidade reais do dispositivo, este deverá ser testado pela população-alvo, etapas futuras do projeto em questão.

\section{CONCLUSÃO}

A confecção do SL, tendo resultados satisfatórios quanto aos objetivos propostos, se deu pela redução dos custos visando a um produto que alcance o mercado sem descuidar da segurança e do conforto para o usuário. $\mathrm{Na}$

\section{REFERÊNCIAS}

1. Resnikoff S, Pascolini D, Etya'ale D, Kocur I, Pararajasegaram R, Pokharel Gp, Mariotti Sp. Global data on visual impairment in the year 2002. Bull World Health Organ, 2004;82(11):844-51. DOI: http://dx.doi. org/10.1590/S0042-96862004001100009.

2. World Health Organization (WHO). Visual impairment and blondness [cited 2013 July 01]. Available from: www.who. int/mediacentre/factsheets/fs282/en/.

3. Instituto Brasileiro De Geografia e Estatística - IBGE. Censo demográfico. Características gerais da população, religião e pessoas com deficiência. Brasília; 2010.

4. Temporini ER, Kara-José N. A perda da visão: estratégias de prevenção. Arq Bras Oftalmol. 2004;67(4):597-601. DOI: http://dx.doi.org/10.1590/S0004-27492004000400007.

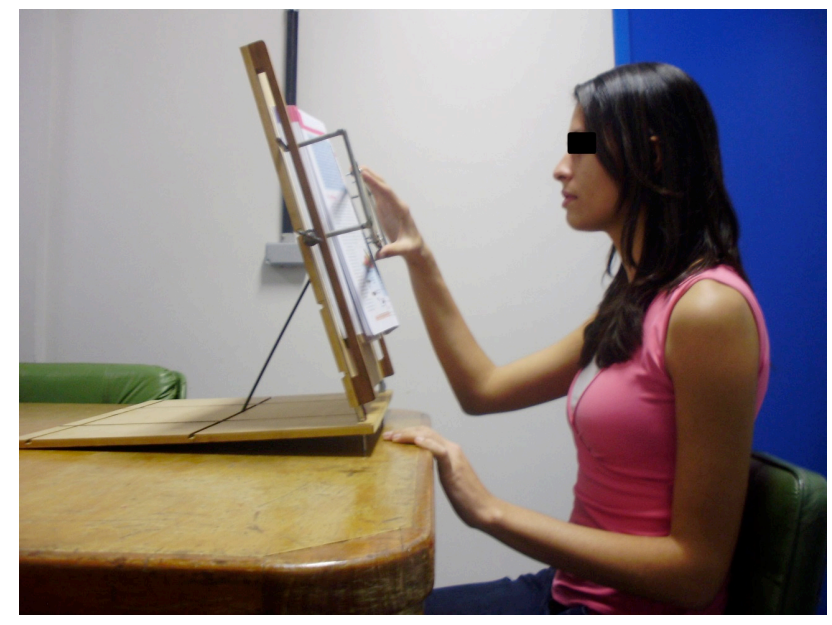

Figura 4 - Suporte de leitura em uso

medida em que a pesquisa se desenvolveu, mostrou formas de utilização viáveis, ressaltando a importância de elaborar um equipamento com qualidades estéticas que podem contribuir para melhorar o estado de aceitação do produto por meio de ferramentas de design computacional e testes com materiais. O processo de observação do cotidiano escolar, somado à manutenção de um diálogo constante com a comunidade e um grupo interdisciplinar, possibilitou a confecção de um objeto de acesso à leitura de rotina e o aprendizado de estudantes no ambiente escolar, com praticidade e conforto para o usuário. Será fundamental dar continuidade aos testes do SL para garantir que o equipamento atenda às necessidades propostas pelo trabalho.
5. Haddad M, Sampaio MW, Kara-José N. Auxílios para baixa visão. São Paulo: Editora Laramara; 2001.

6. Bonatti FAS. Desenvolvimento de equipamento de auxílio à visão subnormal. Arq Bras Oftalmol. 2006;69(2):221-6. DOI: $10.1590 /$ S0004-27492006000200016.

7. Carvalho KMM, Gasparetto MERF, Venturini NHB. Visão subnormal - orientação ao professor do ensino regular. Campinas: Unicamp; 1992.

8. Souza AGM, Albuquerque RC. A atuação da terapia ocupacional na intervenção precoce de crianças com baixa visão utilizando a estimulação visual. Temas Desenvolv. 2005;13(78):29-34.

9. Veitzman S. Visão subnormal. Rio de Janeiro: Cultura Médica; 2000. (Coleção de Manuais Básicos CBO). 
10. Bersch R. Introduçãoà tecnologia assistiva. PortoAlegre:CEDICentro Especializado em Desenvolvimento Infantil; 2008.

11. Toledo C, Paiva APG, Camilo GB, Maior MRS, Leite ICG, Guerra MR. Detecção precoce de deficiência visual e sua relação com o rendimento escolar. Rev Assoc Med Bras. 2010;56(4):415-9. DOI: http://dx.doi.org/10.1590/S010442302010000400013.

12. Bezerra CP, Pagliuca LMF. As relações interpessoais do adolescente visual na escola. Rev Gaúcha Enfermagem. 2007;28(3):315-23.

13. Bidarra J, Boscarioli C, Peres SM. Software x lupa - um ampliador de tela para auxílio na educação de alunos com baixa visão. Rev Bras Educ Espec. 2011;17(1):151-72. DOI: http://dx.doi.org/10.1590/S1413-65382011000100011.

14. Gasparetto MERF. Orientações ao professor e à comunidade escolar referente ao aluno com baixa visão. In: Sampaio MW et al., organizadores. Baixa visão e cegueira: os caminhos para a reabilitação, a educação e a inclusão. Rio de Janeiro: Cultura Médica/Guanabara Koogan; 2010. p.347-60.

15. Ferroni MCC, Gasparetto MERF. Escolares com baixa visão: percepção sobre as dificuldades visuais, opinião sobre as relações com comunidade escolar e o uso de recursos de tecnologia assistiva nas atividades cotidianas. Rev Bras Educ Espec. 2012;18(2):301-18. DOI: http:// dx.doi.org/10.1590/S1413-65382012000200009.

16. Massambani O, Millán RDS, Schor HHR, Guimarães PG. Rede FORTEC de tecnologia assistivas. Catálogo Fortec de tecnologia assistivas. In: Fórum Nacional de Gestores de Inovação e Transferência de Tecnologia. São Paulo, SP; 2011.

17. Bonatti FAZ, Bonatti JA, Sampaio MW, Haddad MAO, Souza PRM, Kara-José N. Avaliação de pacientes utilizando equipamento inovador de auxílio à visão subnormal. Arq Bras Oftalmol. 2008;71(3):385-8. DOI: http://dx.doi. org/10.1590/S0004-27492008000300015.

18. Bonatti JA, Bonatti FAS, Santos MCL, Carricondo PC, Kara-José N. Development of an aspheric 22-diopter $50-\mathrm{mm}$ diameter magnifier. Arq Bras Oftalmol. 2008;71(2):234-37. DOI: http://dx.doi.org/10.1590/S000427492008000200018 .

19. Freitas KPN, Barros SS, Ângelo RCO, Uchôa EPBL. Lombalgia ocupacional e a postura sentada: efeitos da cinesioterapia laboral. Rev Dor (São Paulo). 2011;2(4):308-13. DOI: http://dx.doi.org/10.1590/S180600132011000400005 .

Recebido para publicação: 16.04.14

Aceito para publicação: 27.02.15 